\title{
Técnica de remodelamento com uso de stents em T e molas para tratamento de aneurisma renal complexo
}

\author{
Remodeling technique using T-stenting and coils to treat complex renal aneurysm \\ Patrick Bastos Metzger ${ }^{1}$ (D), Kamilla Rosales $\operatorname{Costa}^{1}$ (D), Simone Lessa Metzger ${ }^{2}$
}

\begin{abstract}
Resumo
O aneurisma da artéria renal é uma condição rara, que vem sendo cada vez mais diagnosticada devido ao uso mais amplo da angiotomografia. Descrevemos um caso de aneurisma da artéria renal tipo II complexo em uma paciente com hipertensão arterial sistêmica e doença renal crônica não dialítica. O tratamento estabelecido foi o reparo endovascular através da combinação da técnica de remodelamento com stents em Te molas, para a preservação dos ramos arteriais renais. Foram obtidos resultados arteriográicos satisfatórios e boa evolução clínica.
\end{abstract}

Palavras-chave: aneurisma; artéria renal; procedimentos endovasculares.

\begin{abstract}
Renal artery aneurysm is a rare condition that is being diagnosed with increasing frequency because of wider use of angiotomography. We describe a case of complex type II renal artery aneurysm in a patient with systemic arterial hypertension and non-dialysis chronic kidney disease. The treatment performed was endovascular repair using the remodeling technique with T-stenting and coils to preserve the renal arterial branches, obtaining satisfactory arteriographic results and good clinical outcomes.
\end{abstract}

Keywords: aneurysm; renal artery; endovascular procedures.

Como citar: Metzger PB, Costa KR, Metzger SL. Técnica de remodelamento com uso de stents em T e molas para tratamento de aneurisma renal complexo. J Vasc Bras. 2021;20:e20200141. https://doi.org/10.1590/1677-5449.200141

'Escola Bahiana de Medicina e Saúde Pública - EBMSP, Salvador, BA, Brasil.

${ }^{2}$ Obra Sociais Irmã Dulce - OSID, Hospital Santo Antônio, Salvador, BA, Brasil.

Fonte de financiamento: Nenhuma.

Conflito de interesse: Os autores declararam não haver conflitos de interesse que precisam ser informados.

Submetido em: Julho 27, 2020. Aceito em: Fevereiro 12, 2021.

O estudo foi realizado no Hospital Geral Roberto Santos (HGRS), Salvador, BA, Brasil. 


\section{INTRODUÇÃO}

$\mathrm{O}$ aneurisma de artéria renal (AAR) constitue uma afecção incomum, tendo uma prevalência de menos de 0,3 a $1 \%$ da população. Atualmente, devido ao maior uso de métodos de imagem como a ultrassonografia com Doppler e a angiotomografia computadorizada (ATC), o número de diagnósticos vem aumentando ${ }^{1,2}$.

As indicações para o tratamento e a escolha da técnica ainda são controversas devido ao número reduzido de estudos disponíveis, por se tratar de uma doença rara, o que dificulta a realização de ensaios clínicos randomizados ${ }^{2,3}$. A cirurgia aberta pode ser realizada através da excisão do aneurisma com reconstrução ou nefrectomia ${ }^{2}$. Com o avanço da técnica endovascular, o tratamento do AAR através do reparo percutâneo tornou-se possível, proporcionando uma redução do trauma cirúrgico e do tempo de internação pós-operatória, bem como da morbidade, quando comparada ao tratamento cirúrgico aberto ${ }^{1,4}$. No entanto, o tratamento dos AAR complexos, em que a preservação dos ramos para a irrigação dos polos renais emergem do aneurisma, continua sendo um desafio terapêutico ${ }^{1-4}$. Novas técnicas e materiais derivados da neurointervenção parecem alternativas promissoras para o tratamento desses AAR complexos.

Relatamos o tratamento endovascular de um AAR tipo II complexo, envolvendo a bifurcação da artéria renal principal, com saída de um grande ramo polar para o polo médio do aneurisma através da combinação da técnica de remodelamento com stents em $\mathrm{T}$ e molas. O objetivo foi preservar os ramos arteriais de cada polo renal em uma paciente com doença renal crônica (DRC) não dialítica e hipertensão arterial sistêmica (HAS) refratária. A paciente consentiu com a publicação do caso clínico e de suas imagens. O estudo foi aprovado pelo Comitê de Ética local (número do parecer 4.451.756).

\section{RELATO DE CASO}

Mulher de 55 anos, hipertensa, dislipidêmica e portadora de DRC estágio III, em acompanhamento com equipe de cardiologia devido a HAS de difícil controle, em uso de quatro drogas anti-hipertensivas. Foi identificado, durante investigação com Doppler de artérias renais, um volumoso aneurisma em hilo renal direito. Realizou-se ATC de abdome, que demonstrou um AAR tipo II de $3,4 \times 2,8 \mathrm{~cm}$, envolvendo a bifurcação da artéria renal principal, que irrigava os polos superior e inferior e um volumoso ramo que nutria o polo médio renal com saída posterior do aneurisma (Figura 1). A paciente apresentava clearance de creatinina de $41 \mathrm{~mL} / \mathrm{min} / 1,73 \mathrm{~m}^{2}$.

Após criterioso estudo angiotomográfico renal e estudo das possibilidades de tratamento para maior
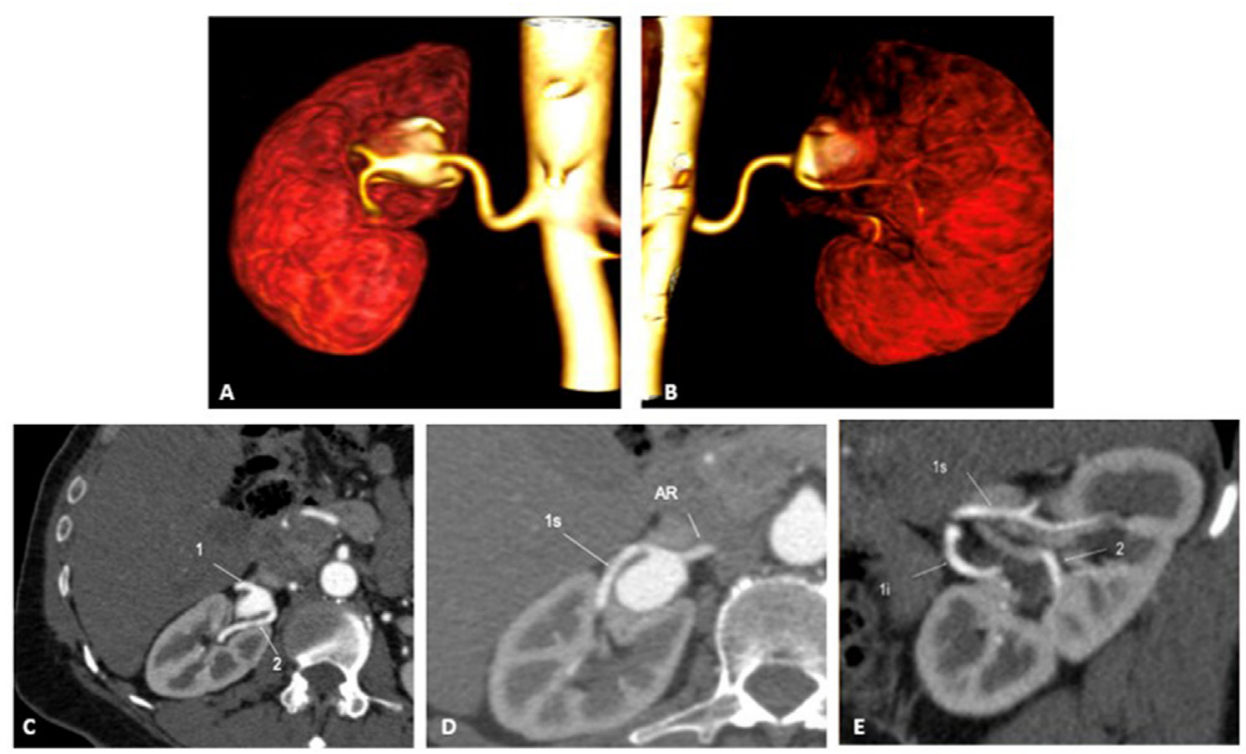

Figura 1. (A) Angiotomografia com reconstrução volumétrica de aneurisma renal tipo II visão anteroposterior, demonstrando aneurisma renal tipo II acometendo a bifurcação renal; (B) Angiotomografia com reconstrução volumétrica de aneurisma renal tipo II visão posteroanterior, demonstrando aneurisma renal tipo II com ramo nutridor no polo renal médio saindo do aneurisma renal tipo II; (C) Corte angiotomográfico multiplanar demonstrando em 1 - artéria renal principal próximo a bifurcação superior e inferior; em 2 - artéria nutridora do polo médio saindo do aneurisma; (D) Corte angiotomográfico multiplanar demonstrando em 1s - ramo nutridor do polo renal superior; AR - artéria renal principal; (E) Corte angiotomográfico multiplanar demonstrando em 1s - ramo nutridor do polo renal superior; $1 \mathrm{i}$ - ramo nutridor do polo renal inferior; 2 - ramo nutridor do polo renal médio. 
preservação do parênquima, optou-se pela realização de tratamento endovascular utilizando a técnica de remodelamento com stents em $\mathrm{T}$ (t-stenting) e molas para preservação das artérias renais nutridoras. $\mathrm{O}$ tratamento foi realizado utilizando introdutor longo 6 Fr Destination (Terumo Medical, Somerset, EUA) com cateterização da artéria renal direita com uso de cateter cobra 2 e fio guia hidrofílico $0,035 \times 260 \mathrm{~cm}$ (Terumo Medical, Somerset, EUA). Após arteriografia renal com melhor visualização da bifurcação da artéria renal principal (Figura 2A), foi cateterizado o ramo superior da artéria renal principal com uso de fio guia 0,035 x 260 amplatz (Boston Scientific, Minneapolis, EUA), sendo implantado o stent Palmaz Genesis Pro 5 x 39 mm (Cordis Corporation, Warren, EUA) (Figura 2B) justa bifurcação. Na sequência, foi cateterizada a malha do stent Palmaz Genesis Pro (Cordis Corporation, Warren, EUA) com uso de fio guia Pt2 0,014 x $180 \mathrm{~cm}$ moderado suporte (Boston Scientific, Minneapolis, EUA) e realizada a cateterização do ramo nutridor do polo médio, sendo implantado um stent Palmaz Blue $3 \times 18 \mathrm{~mm}$ (Cordis Corporation, Warren, EUA), a partir da malha do stent Genesis Pro para a artéria nutridora do polo médio. Foi realizada, então, cateterização utilizando microcateter Progreat 2.4F (Terumo Medical, Somerset, EUA), entre as malhas do stent, e realizada a liberação de 6 micromolas hidrocoloidais AZUR-18 x 12mm x $20 \mathrm{~cm}$ destacáveis (Terumo Medical, Somerset, EUA) sobre o saco aneurismático (Figura 2C). A arteriografia de controle demonstrou preservação dos ramos nutridores renais e embolização do saco aneurismático renal (Figura 2D).

A paciente evoluiu satisfatoriamente no pósoperatório, tendo alta no $3^{\circ}$ dia, sem elevação das escorias nitrogenadas ou hematúria, em uso de dupla antiagregação. Realizou ATC no acompanhamento ambulatorial de 3 meses após o procedimento, demonstrando parênquima renal sem isquemia e com todos os ramos nutridores preservados (Figura 3A e 3B). Atualmente, encontra-se em acompanhamento há 3 anos com boa evolução clinica, laboratorial e de imagem. Durante esse período de acompanhamento, não houve a necessidade de terapia substutiva renal, assim como a paciente permaneceu em uso de quatro classes de drogas anti-hipertensivas, mantendo controle pressórico adequado.
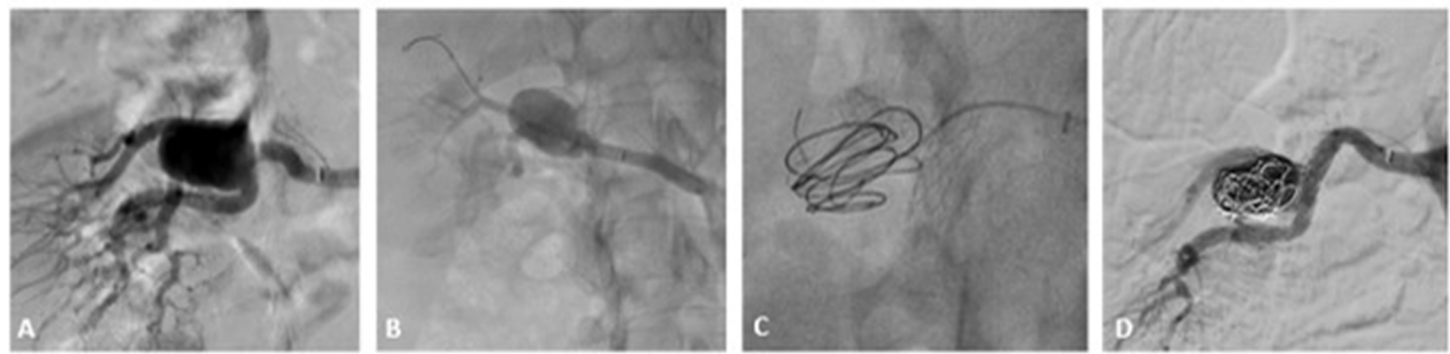

Figura 2. (A) Arteriografia renal demonstrando aneurisma renal tipo II complexo - pré-tratamento; (B) Posicionamento do $1^{\circ}$ stent justa bifurcação da artéria renal principal; (C) Posicionameto dos stents em T e inicio da embolização vias malhas do stent; (D) Arteriografia de controle final demonstrando ramos nutridores pérvios e embolização do aneurisma renal.
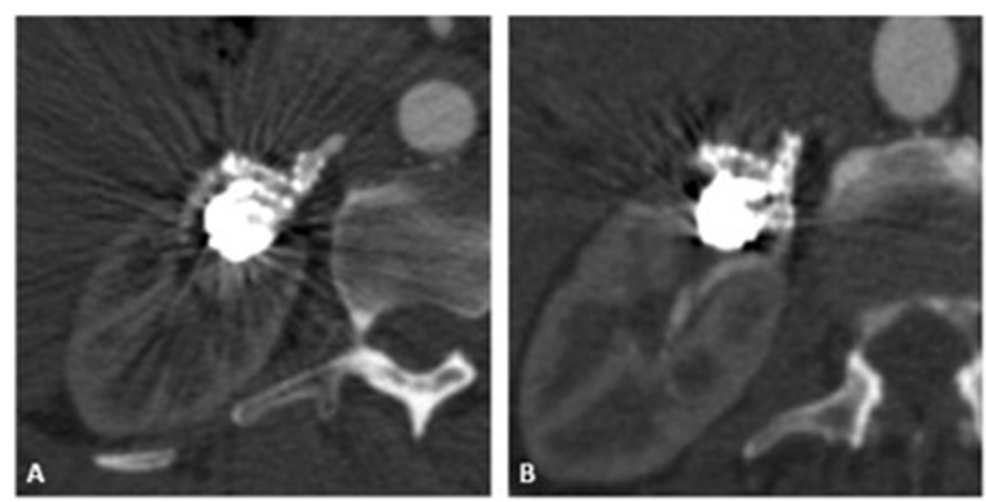

Figura 3. (A) Controle angiotomográfico com 3 meses demonstrando perviedade do ramo nutridor do polo médio; (B) Controle angiotomográfico com 3 meses demonstrando perviedade do ramo nutridor do polo superior e inferior. 


\section{DISCUSSÃO}

Os aneurismas de artérias viscerais (AAV) são raros, presentes em menos de $1 \%$ da população total. O AAR é um dos tipos menos frequentes de AAV, correspondendo a cerca de 15 a $22 \%{ }^{4}$. Estudos realizados através de autópsia indicam uma incidência de AAR de $0,1 \%$. No entanto, os estudos realizados através da arteriografia renal evidenciam uma incidência que varia de 0,3 a $1 \%{ }^{1,2,5}$. Essa doença é mais comum no sexo feminino, devido à forte associação com a fibrodisplasia muscular renal ${ }^{1,6}$, porém também está relacionada a aterosclerose, infecções, trauma, doença de Kawasaki, síndrome de Marfan, displasias vasculares e poliarterite nodosa $(\mathrm{PAN})^{1,3}$. A maioria dos pacientes é assintomática, mas alguns podem apresentar sintomas como HAS, isquemia renal, hematúria ou dor em flanco, mas a relação é pouco estabelecida. As complicações envolvem hipertensão renal vascular, trombose e embolia renal, infarto renal e ruptura ${ }^{7}$. A ruptura, apesar de um fenômeno relativamente pouco frequente (5 a 10\%), associa-se a altas taxas de mortalidade $(80 \%)^{1}$. Em nosso caso, a descoberta do AAR foi incidental e motivada pela a investigação da HAS refratária de etiologia renovascular.

Trabalhos como os de Barros et al. ${ }^{1}$ e Abath et al. ${ }^{7}$ demonstram que os AAR tipo II são os mais comuns quando comparados com os demais tipos. Esse tipo de aneurisma é definido pela literatura como uma lesão desafiadora, principalmente quando adjacente a bifurcações arteriais, tendo o tratamento cirúrgico aberto (endoaneurismorrafia, nefrectomia ou autotransplante) como opção de tratamento já estabelecido há décadas ${ }^{6,7}$. Estudos mais recentes recomendam o reparo endovascular através do uso das técnicas de remodelamento com uso de stent associado ao uso de molas ou de molas e agentes embolizantes líquidos, com o objetivo de preservar os vasos renais nativos quando situados em artéria renal principal ou envolvendo bifurcações nos AAR saculares de colo largo ${ }^{1,2,7}$. Ainda assim, essas lesões são um desafio para esse tipo de reparo devido à sua difícil anatomia, exigindo técnicas mais complexas ${ }^{1,7}$. Da mesma forma, os AAR tipo II fusiformes apresentam grande dificuldade de tratamento percutâneo atualmente ${ }^{1,7}$. Apesar disso, o reparo endovascular tem eficácia comprovada em 89,7 a $98 \%$ dos casos, proporcionando uma redução da morbidade, do tempo de operação e do tempo de internação pós-operatória, além de menor trauma cirúrgico ${ }^{1,4,7}$.

As indicações para o tratamento do AAR permanecem ainda incertas na literatura, porém há consenso de que existe indicação de tratamento em pacientes sintomáticos, em aneurismas volumosos, com embolizações renais, aneurisma em mulheres grávidas ou em idade fértil e aneurismas $>2,5 \mathrm{~cm}^{1,2,6-12}$.

Até o desenvolvimento das técnicas de remodelamento com stent, os aneurismas saculares de colo largo (proporção saco aneurismático:colo $<2$ ) não podiam ser tratados por embolização seletiva, tendo um risco significativo de oclusão do vaso principal causada pela migração da mola ou do agente embolizante líquido para a artéria desprotegida. Com o surgimento desta técnica, um stent é implantado na artéria principal no colo do aneurisma, enquanto as micromolas ou outros agentes embolizantes são depositados no aneurisma através da malha do stent, que tem um papel de suporte, evitando, assim, a migração inadvertida dos materiais emboligênicos, além de contribuir para alterar os parâmetros hemodinâmicos localmente, redirecionando o fluxo e fornecendo um substrato para endotelização naquela área, o que diminui a chance de recanalização de aneurismas a longo prazo ${ }^{1,2,7-12}$. Os aneurismas saculares com colo estreito (proporção saco aneurismático:colo > 2) são candidatos para embolização primária do saco aneurismático com molas ou agentes embólicos líquidos ${ }^{1,9-12}$.

A utilização de stents, empregados primariamente em procedimentos de neurointervenção, como o Solitaire (Medtronic, Minneapolis, EUA), apresenta grande navegabilidade e flexibilidade, passando no interior de microcateteres. Inclusive, o stent apresenta a vantagem de poder ser reposicionado mesmo após ter sido completamente liberado. Porém, mais estudos com resultados a longo prazo da utilização desses stents em AAR são necessários ${ }^{10}$. Em nosso caso, não havia a disponibilidade para o uso desses stents.

Os avanços tecnológicos recentes envolvem técnicas endovasculares com stents moduladores de fluxo. Esses stents apresentam múltiplas camadas especificamente desenhadas para reduzir a velocidade de fluxo no interior do saco aneurismático, promovendo trombose e manutenção do fluxo na artéria principal e seus ramos. Eles são amplamente utilizados na neurointervenção, mas sua utilização em vasos periféricos ainda carece de evidências, com pequenas séries e relatos ${ }^{9,10}$.

No caso relatado, observamos um grande ramo nutridor do polo médio saindo do AAR tipo II, em uma paciente com DRC estagio III, havendo a necessidade de preservação do parênquima renal. Após a análise pormenorizada da ATC em diversas reconstruções e cálculos das artérias aferentes e eferentes do aneurisma, optamos por realizar a preservação da artéria renal utilizando stents de nitinol balão expansíveis, utilizando as malhas do stent como suporte para a preservação do ramo nutridor do polo médio e como apoio para as molas, evitando a migração delas. Após o implante dos stents, preenchemos o saco aneurismático com 
molas hidrocoloidais no intuito de embolizar o saco aneurismático. O controle arteriográfico intraoperatório e o controle angiotomográfico 3 meses após procedimento demonstraram perviedade dos ramos tratados, ausência de isquemia renal e embolização do saco aneurismático.

A combinação de técnicas que foi realizada para essa paciente é de complexa execução, necessitando de um estudo angiotomográfico rigoroso prévio para avaliação da anatomia e o adequado planejamento do procedimento. Dessa forma, quando a anatomia vascular permitir, é possível reparar AAR tipo II complexo preservando os vasos nativos da topografia renal, como fora realizado neste caso. Não há dúvida de que a presença de uma função renal limítrofe associada a um AAR complexo imponha a necessidade de preservação renal, sendo essa técnica de remodelamento com stents em $\mathrm{T}$ e molas bem indicada nesses casos. Atualmente os conhecimentos relacionados a novos materiais e técnicas derivados da neurointervenção tornam o tratamento dos AAR complexos exequível e efetivo, devendo influenciar na escolha terapêutica.

\section{REFERÊNCIAS}

1. Barros KJF, Metzger PB, Rossi FH, et al. Técnicas e táticas no tratamento endovascular do aneurisma da artéria renal. Rev Bras Cardiol Invasiva. 2014;22(1):64-72. http://dx.doi.org/10.1590/0104-1843000000012.

2. Kitzing B, Vedelago J, Bajic N, Lai G, Waugh R. Stent-assisted coil embolization of a wide-necked renal artery aneurysm. J Radiol Case Rep. 2010;4(4):20-4. http://dx.doi.org/10.3941/jrcr.v4i4.360. PMid:22470722.

3. Goy J-J, Tinguely F, Poncioni L, Berger A, Stauffer J-C. Aneurysm of the renal artery in a patient with the Marfan syndrome, treated by stenting and coils implantation. Catheter Cardiovasc Interv. 2007;69(5):701-3. http://dx.doi.org/10.1002/ccd.21089. PMid:17330268.

4. Vallina-Victorero Vazquez MJ, Lorenzo FV, Salgado AA, et al. Endovascular treatment of splenic and renal aneurysms. Ann Vasc Surg. 2009;23(2):258.e13-7. http://dx.doi.org/10.1016/j. avsg.2008.05.018. PMid:18990544.

5. Tham G, Leifekelund, Herrlin K, Lindstedt EL, Olin T, Bergentz S-E. Renal artery aneurysms. Natural history and prognosis. Ann Surg. 1983;197(3):348-52. http://dx.doi.org/10.1097/00000658198303000-00016. PMid:6830341.

6. Tsilimparis N, Reeves JG, Dayama A, Perez SD, Debus ES, Ricotta J 2 nd. Endovascular vs open repair of renal artery aneurysms: outcomes of repair and long-term renal function. J Am Coll Surg. 2013;217(2):263-9. http://dx.doi.org/10.1016/j.jamcollsurg.2013.03.021. PMid:23769185.

7. Abath C, Andrade G, Cavalcanti D, Brito N, Marques R. Complex renal artery aneurysms: liquids or coils? Tech Vasc Interv Radiol.
2007;10(4):299-307. http://dx.doi.org/10.1053/j.tvir.2008.03.009. PMid:18572145.

8. Rundback JH, Rizvi A, Rozenblit GN, et al. Percutaneous stentgraft management of renal artery aneurysms. J Vasc Interv Radiol. 2000;11(9):1189-93. http://dx.doi.org/10.1016/S1051-0443(07)613621. PMid:11041477.

9. Metzger PB, Kambara AM, Barbato HA, Rossi FH, Izukawa NM. Endovascular approach of a patient with bilateral renal artery fibrodysplasia associated with a massive renal aneurysm. Rev Bras Cardiol Invasiva. 2015;23(2):145-7. http://dx.doi.org/10.1016/j. rbci.2015.12.015.

10. Belczak SQ. Tratamento de aneurismas de artéria esplênica e renal no mesmo tempo operatório: relato de caso e revisão. J Vasc Bras. 2020;19:e20200004. http://dx.doi.org/10.1590/1677-5449.200004.

11. Cardozo MA, Lichtenfels E, Erling N Jr, Raupp E, Tarasconi DP. Tratamento endovascular de aneurisma da artéria renal por em-bolização com micromolas preservando o fluxo sangüíneo renal: relato de caso. J Vasc Bras. 2007;6(2):167-70. http://dx.doi. org/10.1590/S1677-54492007000200012.

12. Brito LCM, Martins JT, Passos E, Santos AJ, Gama RAD, Furlani GX. Tratamento de aneurisma da artéria renal por embolização e técnica de remodelamento de colo: relato de caso. J Vasc Bras. 2011;10(2):181-4. http://dx.doi.org/10.1590/S1677-54492011000200016.

Correspondência Patrick Bastos Metzger Escola Bahiana de Medicina e Saúde Pública - EBMSP Rua Professor Diógenes Rebouças - Pituba CEP 41830-570 - Salvador (BA), Brasil Tel.: (71) 99977-6356 E-mail: patrickvascular@gmail.com

Informações sobre os autores PBM - Doutor em Medicina, Universidade de São Paulo (USP); Professor Adjunto, Escola Bahiana de Medicina e Saúde Pública (EBMSP); Cirurgião Vascular e Endovascular, Sociedade Brasileira de Angiologia e Cirurgia Vascular (SBACV); Radiologista Intervencionista, Colégio Brasileiro de Radiologia (CBR).

KRC - Acadêmica, $9^{\circ}$ semestre de Medicina, Escola Bahiana de Medicina e Saúde Pública (EBMSP).

SLM - Geriatra, Sociedade Brasileira de Geriatria e Gerontologia

(SBGG); Professora Auxiliar, Escola Bahiana de Medicina e Saúde

Pública (EBMSP); Professora Assistente, Universidade Salvador (UNIFACS); Preceptora da Residência de Geriatria, Obras Sociais Irmã Dulce (OSID).

Contribuições dos autores Concepção e desenho do estudo: PBM Análise e interpretação dos dados: PBM Coleta de dados: PBM Redação do artigo: PBM, KRC, SLM Revisão crítica do texto: PBM Aprovação final do artigo: PBM, KRC, SLM Análise estatística: PBM, SLM Responsabilidade geral pelo estudo: PBM, KRC, SLM

*Todos os autores leram e aprovaram a versão final submetida ao J Vasc Bras. 